Bull. Korean Math. Soc. 50 (2013), No. 1, pp. 83-96

http://dx.doi.org/10.4134/BKMS.2013.50.1.083

\title{
TORSION POINTS OF ELLIPTIC CURVES WITH BAD REDUCTION AT SOME PRIMES II
}

\author{
MASAYA YASUdA
}

\begin{abstract}
Let $K$ be a number field and fix a prime number $p$. For any set $S$ of primes of $K$, we here say that an elliptic curve $E$ over $K$ has $S$-reduction if $E$ has bad reduction only at the primes of $S$. There exists the set $B_{K, p}$ of primes of $K$ satisfying that any elliptic curve over $K$ with $B_{K, p}$-reduction has no $p$-torsion points under certain conditions. The first aim of this paper is to construct elliptic curves over $K$ with $B_{K, p^{-}}$ reduction and a $p$-torsion point. The action of the absolute Galois group on the $p$-torsion subgroup of $E$ gives its associated Galois representation $\bar{\rho}_{E, p}$ modulo $p$. We also study the irreducibility and surjectivity of $\bar{\rho}_{E, p}$ for semistable elliptic curves with $B_{K, p}$-reduction.
\end{abstract}

\section{Introduction}

Let $E$ be an elliptic curve over a number field $K$. For a prime number $p$, the $p$-torsion points of $E$ are the points of finite order $p$ in the Mordell-Weil group $E(K)$. In [14], we studied the existence of a $p$-torsion points of $E$ which has bad reduction only at some primes, and showed the following [14, Theorem 1.2]. Let $\zeta_{p}$ denote a fixed primitive $p$-th root of unity.

Theorem 1.1. Let $K$ be a number field. Let $p \geq 5$ be a prime number such that the ramification index $e_{\mathfrak{p}}$ satisfies $e_{\mathfrak{p}}<p-1$ for the primes $\mathfrak{p}$ of $K$ over p. Set

$B_{K, p}=\left\{\mathfrak{q}:\right.$ prime of $K$ over a prime $\ell \mid \ell \neq p$ and $\left.\ell^{f} \not \equiv \pm 1 \bmod p\right\}$,

where $f$ is the residue degree of $\mathfrak{q}$. Let $E$ be an elliptic curve over $K$ with $B_{K, p}$-reduction. If $p$ does not divide the class number $h_{K\left(\zeta_{p}\right)}$ of $K\left(\zeta_{p}\right)$, then $E$ has no p-torsion points.

Fix a prime number $p \geq 5$ with $e_{\mathfrak{p}}<p-1$ for the primes $\mathfrak{p}$ of $K$ over $p$. It follows by Theorem 1.1 that $h_{K\left(\zeta_{p}\right)}$ is divisible by $p$ if there exists $E$ over $K$ with $B_{K, p}$-reduction and a $p$-torsion point. The motivation of this paper arises from the question whether we can construct such pairs $(E, K)$. We here focus

Received April 25, 2011; Revised May 7, 2012.

2010 Mathematics Subject Classification. Primary 14H52; Secondary 14G05.

Key words and phrases. reduction of elliptic curves, torsion points, Galois representation. 
on the case where $K$ is a quadratic field. Kamienny [2] and Kenku-Momose [3] classified the possible torsion subgroups of elliptic curves over quadratic fields $K$, and showed that any elliptic curve over $K$ cannot have $p$-torsion points for the primes $p \geq 17$. Hence it is sufficient to consider the cases $p=5,7,11$ and 13. In the cases $p=5$ and 7 , we construct such pairs $(E, K)$ using Kubert's parametrization [5, Table 3] for an infinite family of elliptic curves with a $p$ torsion point. Furthermore, we list such pairs $(E, K)$ and their $h_{K\left(\zeta_{p}\right)}$. For the cases $p=11$ and 13, Jeon, Kim and Lee [1] gave an infinite family of elliptic curves $E$ over quadratic fields $K$ with a $p$-torsion point. On the contrary to the cases $p=5$ and 7 , we show that an elliptic curve from the family given by Jeon, Kim and Lee cannot have $B_{K, p}$-reduction with very high probability.

Let $G_{K}$ denote the absolute Galois group $\operatorname{Gal}(\bar{K} / K)$. The action of $G_{K}$ on the $p$-torsion subgroup $E[p]$ gives its associated Galois representation

$$
\bar{\rho}_{E, p}: G_{K} \rightarrow \operatorname{Aut}(E[p]) \simeq \mathrm{GL}_{2}\left(\mathbb{F}_{p}\right)
$$

modulo $p$. We also study the irreducibility and surjectivity of $\bar{\rho}_{E, p}$ for semistable elliptic curves $E$ with $B_{K, p}$-reduction. Using a part of Serre's results [11], Mazur [7] studied the surjectivity of $\bar{\rho}_{E, p}$ for semistable elliptic curves $E$ over $\mathbb{Q}$. Furthermore, Kraus [4] extended Mazur's work to the case where $K$ is a quadratic field. We then focus on the case where $K$ is a cubic field. We mainly consider the irreducibility of $\bar{\rho}_{E, p}$. If $\bar{\rho}_{E, p}$ is reducible, then we have

$$
\bar{\rho}_{E, p} \sim\left(\begin{array}{cc}
\phi_{1} & * \\
0 & \phi_{2}
\end{array}\right),
$$

where $\phi_{i}: G_{K} \rightarrow \mathbb{F}_{p}^{\times}$is a character for $i=1,2$. For semistable elliptic curves $E$ with good reduction at the primes of $K$ over $p$, we show that each $\phi_{i}$ has a structure of an $\mathcal{O}_{K}$-group scheme of order $p$, where $\mathcal{O}_{K}$ is the ring of integers of $K$. Our main idea is to classify the structure of $\mathcal{O}_{K}$-group schemes of order $p$ for cubic fields $K$ and show that either of $\phi_{i}$ is a trivial character under certain conditions. We obtain the following result on the irreducibility of $\bar{\rho}_{E, p}$.

Theorem 1.2. Let $p$ be an odd prime number. Let $K$ be a cubic field with $\operatorname{gcd}\left(p-1, h_{K}^{+}\right)=1$, where $h_{K}^{+}$is the narrow class number of $K$. Suppose that $p \nmid h_{K\left(\zeta_{p}\right)}$ and $e_{\mathfrak{p}}<p-1$ for the primes $\mathfrak{p}$ of $K$ over $p$. Let $E$ be a semistable elliptic curve over $K$ with $B_{K, p}$-reduction. If $p$ satisfies either of the following conditions, then $\bar{\rho}_{E, p}$ is irreducible.

(A) $p$ is prime in $\mathcal{O}_{K}$.

(B) In the case where $K$ is a totally real cubic field, we have $p \nmid \mathrm{Nm}_{K / \mathbb{Q}}\left(u^{2}-\right.$ 1) or $\mathrm{Nm}_{K / \mathbb{Q}}\left(v^{2}-1\right)$, where $u, v$ are two independent fundamental units of $K$ and $\mathrm{Nm}_{K / \mathbb{Q}}$ denotes the norm map. In the case where $K$ is a complex cubic field, we have $p \nmid \mathrm{Nm}_{K / \mathbb{Q}}(u-1)$, where $u$ is a fundamental unit of $K$ such that $u>0$ with respect to the only one real place of $K$.

Notation. The symbols $\mathbb{Z}$, and $\mathbb{Q}$ denote, respectively, the ring of integers, and the field of rational numbers. For a prime $p$, the finite field with $p$ elements 
is denoted by $\mathbb{F}_{p}$. We denote the $p$-adic integers and the $p$-adic number field by $\mathbb{Z}_{p}$ and $\mathbb{Q}_{p}$. Let $\mathcal{O}_{K}$ denote the ring of integers of a number field $K$. For a prime $\mathfrak{p}$ of $K$, let $\mathcal{O}_{\mathfrak{p}}$ denote the completion of $\mathcal{O}_{K}$ at $\mathfrak{p}, k_{\mathfrak{p}}$ its residue field, and $K_{\mathfrak{p}}$ the field of fractions of $\mathcal{O}_{\mathfrak{p}}$.

\section{Preliminaries}

In this section, we consider the property of elliptic curves with $B_{K, p}$-reduction and a $p$-torsion point. We also review families of elliptic curves with a $p$-torsion point.

\subsection{Elliptic curves with $B_{K, p}$-reduction and a $p$-torsion point}

Let $K$ be a number field. Fix a prime number $p \geq 5$ with $e_{\mathfrak{p}}<p-1$ for the primes $\mathfrak{p}$ of $K$ over $p$. If there exists an elliptic curve with $B_{K, p}$-reduction and a $p$-torsion point, it follows by Theorem 1.1 that $p \mid h_{K\left(\zeta_{p}\right)}$ and hence $A_{K\left(\zeta_{p}\right)} \neq 0$, where $A_{F}$ denotes the $p$-part of the ideal class group of a number field $F$. Furthermore, we can see the following property.

Let $E$ be an elliptic curve over $K$ with $B_{K, p}$-reduction and a $p$-torsion point $P$. Using the Weil-pairing $e_{p}: E[p] \times E[p] \rightarrow \mu_{p}$, we define a map $\psi: E[p] \rightarrow \mu_{p}$ by $Q \mapsto e_{p}(P, Q)$, where $\mu_{p}$ denotes the set of the $p$-th root of unity. Since the point $P$ is rational over $K$, this map gives an exact sequence

$$
0 \longrightarrow \mathbb{Z} / p \mathbb{Z} \longrightarrow E[p] \stackrel{\psi}{\longrightarrow} \mu_{p} \longrightarrow 0
$$

of $G_{K}$-modules, where $\mathbb{Z} / p \mathbb{Z}$ denotes the constant $G_{K}$-module generated by $P$. Let $L=K(E[p])$ denote the field generated by the points of $E[p]$. By [14, Poof of Theorem 1.2], we may assume that $L$ is an unramified extension of $K\left(\zeta_{p}\right)$ of degree $p$. The representation $\bar{\rho}_{E, p}$ induces the representation

$$
\rho: \operatorname{Gal}(L / K) \rightarrow \mathrm{GL}_{2}\left(\mathbb{F}_{p}\right)
$$

We note that $\rho$ is faithful and of the form $\left(\begin{array}{cc}1 & * \\ 0 & \omega\end{array}\right)$ by the exact sequence $(1)$ where

$$
\omega: \Delta \rightarrow \mathbb{F}_{p}^{\times}, \quad \Delta=\operatorname{Gal}\left(K\left(\zeta_{p}\right) / K\right)
$$

denotes the cyclotomic character defined by $\sigma\left(\zeta_{p}\right)=\zeta_{p}^{\omega(\sigma)}$ for every $\sigma \in \Delta$. We see that $\rho$ does not split by the assumption $L \neq K\left(\zeta_{p}\right)$, and hence the group $\operatorname{Gal}\left(L / K\left(\zeta_{p}\right)\right)$ is isomorphic to the subgroup of $\mathrm{GL}_{2}\left(\mathbb{F}_{p}\right)$ consisting of all matrices of the form $\left(\begin{array}{ll}1 & k \\ 0 & 1\end{array}\right)$ under the representation $\rho$. We now consider the action of $\Delta$ on $\operatorname{Gal}\left(L / K\left(\zeta_{p}\right)\right)$ by conjugation in $\operatorname{Gal}(L / K)$. Consider $\Delta$ as a subgroup of $\mathbb{F}_{p}^{\times}$under the cyclotomic character $\omega$ and fix $a \in \Delta \subset \mathbb{F}_{p}^{\times}$. Since conjugating $\left(\begin{array}{ll}1 & k \\ 0 & 1\end{array}\right)$ by $a \in \mathbb{F}_{p}^{\times}$gives $\left(\begin{array}{cc}1 & k / a \\ 0 & 1\end{array}\right)$, we see that $a \in \Delta \subset \mathbb{F}_{p}^{\times}$acts on $\operatorname{Gal}\left(L / K\left(\zeta_{p}\right)\right)$ as multiplication by $a^{-1}$. Then we have $A_{K\left(\zeta_{p}\right)}^{\omega^{-1}} \neq 0$, where $R^{\omega^{i}}$ denotes the $\omega^{i}$-eigenspace of a $\mathbb{Z}_{p}[\Delta]$-module $R$ as in the notation of $[13, \S 6.3]$. 


\subsection{Families of elliptic curves with a $p$-torsion point}

2.2.1. The cases $\boldsymbol{p}=\mathbf{5}$ and $\mathbf{7}$. Let $E$ be an elliptic curve over a number field $K$ with a $p$-torsion point $P$. The following result is well-known ([5, 12] for details): For $p=5$, there exists a unique $t \in K \backslash\{0\}$ such that $E$ is isomorphic to an elliptic curve defined by the equation

$$
E_{t}^{(5)}: y^{2}+(1-t) x y-t y=x^{3}-t x^{2}
$$

and the 5 -torsion point $P$ corresponds to $(0,0)$ under the isomorphism. The discriminant of $E_{t}^{(5)}$ is

$$
\Delta\left(E_{t}^{(5)}\right)=t^{5}\left(t^{2}-11 t-1\right) .
$$

Similarly, for $p=7$, there exists a unique $t \in K \backslash\{0,1\}$ such that $E$ is isomorphic to an elliptic curve defined by the equation

$$
E_{t}^{(7)}: y^{2}+\left(1+t-t^{2}\right) x y+\left(t^{2}-t^{3}\right) y=x^{3}+\left(t^{2}-t^{3}\right) x^{2}
$$

and the 7 -torsion point $P$ corresponds to $(0,0)$ under the isomorphism. The discriminant of $E_{t}^{(7)}$ is

$$
\Delta\left(E_{t}^{(7)}\right)=t^{7}(t-1)^{7}\left(t^{3}-8 t^{2}+5 t+1\right) .
$$

2.2.2. The cases $\boldsymbol{p}=\mathbf{1 1}$ and 13. For $p=11$ and 13 , Jeon, Kim and Lee gave an infinite family of elliptic curves $E_{t}^{(p)}, t \in \mathbb{Q}$ over quadratic fields $\mathbb{Q}\left(\sqrt{d_{p}}\right)$ having a $p$-torsion point as follows (see [1, Section 3] for details): For $p=11$, set

$$
\left\{\begin{array}{l}
b_{11}=-\frac{1}{4} t(t-1)\left(t^{2}+1-\sqrt{d_{11}}\right)\left(t^{3}+t-2-t \sqrt{d_{11}}\right) \\
c_{11}=\frac{1}{2} t(t-1)\left(t^{2}+1-\sqrt{d_{11}}\right), \\
d_{11}=t^{4}+2 t^{2}-4 t+1 .
\end{array}\right.
$$

On the other hand, for $p=13$, set

$$
\left\{\begin{aligned}
b_{13} & =-\frac{t\left(t^{4}-t^{2}+t+1-(t-1) \sqrt{d_{13}}\right)\left(t^{3}+t^{2}+1-\sqrt{d_{13}}\right)\left(t^{4}+t^{3}+t+2-t \sqrt{d_{13}}\right)}{4\left(t^{3}+t^{2}-1-\sqrt{d_{13}}\right)}, \\
c_{13} & =-\frac{t\left(t^{3}+t^{2}+1-\sqrt{d_{13}}\right)\left(t^{4}-t^{2}+t+1-(t-1) \sqrt{d_{13}}\right)}{2\left(t^{3}+t^{2}-1-\sqrt{d_{13}}\right)} \\
d_{13} & =t^{6}+2 t^{5}+t^{4}+2 t^{3}+6 t^{2}+4 t+1 .
\end{aligned}\right.
$$

Let $p=11$ or 13 . Let $E_{t}^{(p)}$ be an elliptic curve over a quadratic field $\mathbb{Q}\left(\sqrt{d_{p}}\right)$ defined by the equation

$$
E_{t}^{(p)}: y^{2}+\left(1-c_{p}\right) x y-b_{p} y=x^{3}-b_{p} x^{2}
$$

with $t \in \mathbb{Q}$. Then $E_{t}^{(p)}$ has a $p$-torsion point $(0,0)$.

\section{A construction of elliptic curves with $B_{K, p}$-reduction and a $p$-torsion point (quadratic field case)}

Throughout this section, let $K$ be a quadratic field. 


\subsection{The cases $p=5$ and 7}

In this subsection, we construct pairs $(E, K)$, where $E$ is an elliptic curve over $K$ with $B_{K, p}$-reduction and a $p$-torsion point. To construct such pairs, we give the following result:

Proposition 3.1. Let $p=5$ or 7 . Let $E=E_{t}^{(p)}, t \in \mathcal{O}_{K}$ be an elliptic curve over defined as in $\S 2.2 .1$. Suppose that $E$ has $B_{K, p}$-reduction. Then we have

$$
\begin{cases}t^{2}-11 t-1 \in \mathcal{O}_{K}^{\times} & \text {if } p=5, \\ t^{3}-8 t^{2}+5 t+1 \in \mathcal{O}_{K}^{\times} & \text {if } p=7 .\end{cases}
$$

Proof. For simplicity, we only consider the case $p=7$. Assume $t^{3}-8 t^{2}+5 t+1 \notin$ $\mathcal{O}_{K}^{\times}$. Then there exists a prime $\ell$ dividing $t^{3}-8 t^{2}+5 t+1 \in \mathcal{O}_{K}$. By the equation (3), the elliptic curve $E$ has bad reduction at some prime $\mathfrak{q}$ of $K$ over $\ell$. Since $E$ has $B_{K, p}$-reduction, we may assume $\ell \neq 7$. We note that the solutions of the equation

$$
X^{3}-8 X^{2}+5 X+1=0
$$

define the extension field $K\left(\zeta_{7}+\zeta_{7}^{-1}\right)$ over $K$. Now we consider the following diagram:

$$
\begin{aligned}
& \operatorname{Gal}\left(K\left(\zeta_{7}\right) / K\right) \quad \stackrel{\omega}{\hookrightarrow} \quad(\mathbb{Z} / 7 \mathbb{Z})^{\times} \\
& \sigma \downarrow \quad \downarrow \\
& \operatorname{Gal}\left(K\left(\zeta_{7}+\zeta_{7}^{-1}\right) / K\right) \hookrightarrow(\mathbb{Z} / 7 \mathbb{Z})^{\times} /\{ \pm 1\},
\end{aligned}
$$

where $\omega$ is the cyclotomic character defined as in $\S 2.1$ and $\sigma$ is the restriction map. Let $s \in \operatorname{Gal}\left(K\left(\zeta_{7}\right) / K\right)$ denote the Frobenius map satisfying

$$
\operatorname{Gal}\left(K_{\mathfrak{q}}\left(\zeta_{7}\right) / K_{\mathfrak{q}}\right)=\langle s\rangle \text {. }
$$

Note that we have $\omega(s)=\ell^{f} \in(\mathbb{Z} / 7 \mathbb{Z})^{\times}$, where $f$ denotes the residue degree of $\mathfrak{q}$. Then we can see the following:

$$
\begin{aligned}
& X^{3}-8 X^{2}+5 X+1=0 \bmod \mathfrak{q} \text { has a solution } t \in \mathcal{O}_{K}, \\
\Longrightarrow & X^{3}-8 X^{2}+5 X+1=0 \text { has a solution } t^{\prime} \in \mathcal{O}_{\mathfrak{q}} \text { (by Hensel's lemma), } \\
\Longrightarrow & \sigma(s)=1 \in \operatorname{Gal}\left(K\left(\zeta_{7}+\zeta_{7}^{-1}\right) / K\right) \Longleftrightarrow \ell^{f} \equiv \pm 1 \bmod 7 .
\end{aligned}
$$

This is a contradiction to the fact that $E$ has $B_{K, p}$-reduction. This completes the proof of Proposition 3.1.

We can apply Proposition 3.1 to construct pairs $(E, K)$ as follows: Let $K=\mathbb{Q}(\sqrt{m})$ be a quadratic field, where $m$ is a square-free integer. Set $t=$ $a+b \sqrt{m} \in \mathcal{O}_{K}$ with $2 a, 2 b \in \mathbb{Z}$. Let $0 \neq u=a^{2}-m b^{2} \in \mathbb{Z}$ denote the norm of $t \in \mathcal{O}_{K}$.

3.1.1. The case $\boldsymbol{p}=\mathbf{5}$. By Proposition 3.1, consider the condition

$$
t^{2}-11 t-1 \in \mathcal{O}_{K}^{\times} \Longleftrightarrow \mathrm{Nm}_{K / \mathbb{Q}}\left(t^{2}-11 t-1\right)= \pm 1 .
$$

Since we have

$$
\mathrm{Nm}_{K / \mathbb{Q}}\left(t^{2}-11 t-1\right)=-4 a^{2}-22(u-1) a+u^{2}+123 u+1,
$$


we see that the condition (4) is equivalent to the condition

$$
X^{2}+11(u-1) X-u^{2}-123 u-1= \pm 1
$$

with $X=2 a \in \mathbb{Z}$. Note that the equation (5) can be transformed to the Pell equation

$$
A^{2}-5 B^{2}= \pm 4
$$

with $A=2 X+11(u-1) \in \mathbb{Z}$ and $B=5(u+1) \in \mathbb{Z}$. Let $\epsilon=\frac{1+\sqrt{5}}{2}$ be a fundamental unit of $\mathbb{Q}(\sqrt{5})$. It is well known that the integer solutions of the Pell equation (6) are given by the elements $\pm \epsilon^{n}$ for $n=0,1,2, \ldots$. Since $B \in 5 \mathbb{Z}$, we note that the solutions of the equation (5) corresponds to the elements $\pm \epsilon^{5 n}$ for $n=0,1,2, \ldots$. For example, we have that an integer solution $(A, B)=(-11,-5)$ of the Pell equation (6) corresponds the element $-\epsilon^{5}=$ $-\frac{11+5 \sqrt{5}}{2}$. Therefore we see that a pair $(X, u)=(11,-2)$ satisfies the condition (5).

A computation shows that only the pairs

$$
\begin{aligned}
(X, u)= & (10,-1),(12,-1),(11,-2),(22,-2),(12,10),(-111,10),(10,-12), \\
& (133,-12),(22,121),(-1342,121),(0,-123),(1364,-123)
\end{aligned}
$$

satisfy the condition (5) with $|u|<1000$. For each pair $(X, u)$, we compute a solution $(a, b, m)$ and check whether the elliptic curve $E_{t}^{(5)}, t=a+b \sqrt{m}$ over $K=\mathbb{Q}(\sqrt{m})$ has $B_{K, 5}$-reduction as follows:

- For $(X, u)=(10,-1)$, we have a solution $(a, b, m)=(5,1,26)$. We see that the elliptic curve $E_{t}^{(5)}, t=a+b \sqrt{m}$ has good reduction everywhere over $K=\mathbb{Q}(\sqrt{26})$. Therefore $E_{t}^{(5)}$ has $B_{K, 5}$-reduction.

- For $(X, u)=(11,-2)$, we have a solution $(a, b, m)=\left(\frac{11}{2}, \frac{1}{2}, 129\right)$. We see that the elliptic curve $E_{t}^{(5)}, t=a+b \sqrt{m}$ has bad reduction only at the primes of $K=\mathbb{Q}(\sqrt{129})$ over 2. Therefore $E_{t}^{(5)}$ has $B_{K, 5}$-reduction.

- For $(X, u)=(12,10)$, we have a solution $(a, b, m)=(6,1,26)$. Since the elliptic curve $E_{t}^{(5)}, t=a+b \sqrt{m}$ has bad reduction at the primes of $K=\mathbb{Q}(\sqrt{26})$ over 5 , the elliptic curve $E_{t}^{(5)}$ does not have $B_{K, 5^{-}}$ reduction.

In Table 1, we list the triples $(a, b, m)$ such that the elliptic curve $E_{t}^{(5)}, t=$ $a+b \sqrt{m}$ over $K=\mathbb{Q}(\sqrt{m})$ has $B_{K, 5}$-reduction. Furthermore, for each $(a, b, m)$, we also list $h_{K\left(\zeta_{5}\right)}$ which is computed by using PARI/GP Version 2.4.1 [10]. We note that $h_{K\left(\zeta_{5}\right)}$ is divisible by 5 .

3.1.2. The case $\boldsymbol{p}=7$. As in the case $p=5$, consider the condition

$$
t^{3}-8 t^{2}+5 t+1 \in \mathcal{O}_{K}^{\times} \Longleftrightarrow \mathrm{Nm}_{K / \mathbb{Q}}\left(t^{3}-8 t^{2}+5 t+1\right)= \pm 1
$$


TABLE 1. List of the triples $(a, b, m)$ with $|u|<1000$ such that $E_{t}^{(5)}, t=a+b \sqrt{m}$ over $K=\mathbb{Q}(\sqrt{m})$ has $B_{K, 5}$-reduction $\left(u=a^{2}-m b^{2}\right)$

\begin{tabular}{|c|c|c|}
\hline$(X, u)$ & $(a, b, m)$ & $h_{K\left(\zeta_{5}\right)}$ \\
\hline$(10,-1)$ & $(5,1,26)$ & 40 \\
$(12,-1)$ & $(6,1,37)$ & 5 \\
$(11,-2)$ & $\left(\frac{11}{2}, \frac{1}{2}, 129\right)$ & 10 \\
$(22,-2)$ & $(11,1,123)$ & 160 \\
$(10,-12)$ & $(5,1,37)$ & 5 \\
$(133,-12)$ & $\left(\frac{133}{2}, \frac{1}{2}, 17737\right)$ & 307125 \\
$(-1342,121)$ & $(-671,1,450120)$ & 320 \\
$(0,-123)$ & $(0,1,123)$ & 160 \\
$(1364,-123)$ & $(682,1,465247)$ & 461194240 \\
\hline
\end{tabular}

TABLE 2. List of the triples $(a, b, m)$ with $|u|<1000$ such that $E_{t}^{(7)}, t=a+b \sqrt{m}$ over $K=\mathbb{Q}(\sqrt{m})$ has $B_{K, 7}$-reduction $\left(u=a^{2}-m b^{2}\right)$

\begin{tabular}{|c|c|c|}
\hline$(X, u)$ & $(a, b, m)$ & $h_{K\left(\zeta_{7}\right)}$ \\
\hline$(6,-1)$ & $(3,1,10)$ & 28 \\
$(7,-2)$ & $\left(\frac{7}{2}, \frac{1}{2}, 57\right)$ & 56 \\
$(8,5)$ & $(4,1,11)$ & 28 \\
\hline
\end{tabular}

by Proposition 3.1. A computation shows that $\mathrm{Nm}_{K / \mathbb{Q}}\left(t^{3}-8 t^{2}+5 t+1\right)$ is equal to

$$
8 a^{3}+(20 u-32) a^{2}+\left(-16 u^{2}-86 u+10\right) a+\left(u^{3}+54 u^{2}+4 u+1\right) .
$$

Therefore the condition (7) is equivalent to the condition

(8) $X^{2}+(5 u-8) X^{2}+\left(-8 u^{2}-43 u+5\right) X+\left(u^{3}+54 u^{2}+4 u+1\right)= \pm 1$

with $X=2 a \in \mathbb{Z}$. We see that only the pairs

$$
(X, u)=(2,1),(6,-1),(7,-1),(7,-2),(8,5),(8,6),(9,7)
$$

satisfy the condition (8) with $|u|<1000$. As in the case $p=5$, in Table 2, we list the triples $(a, b, m)$ such that the elliptic curve $E_{t}^{(7)}, t=a+b \sqrt{m}$ over $K=\mathbb{Q}(\sqrt{m})$ has $B_{K, 7}$-reduction. We note that $h_{K\left(\zeta_{7}\right)}$ is divisible by 7 .

Remark. The equation (8) defines a nonsingular projective curve $C$ of genus 1 . It follows from Siegel's Theorem that the set $C(\mathbb{Z})$ of the integer solutions is finite. Therefore, unlike the case $p=5$, there are only finitely many solutions $(X, u) \in \mathbb{Z}^{2}$ of the equation (8). 


\subsection{The cases $p=11$ and 13}

In this subsection, we consider whether we can construct pairs $(E, K)$, where $E=E_{t}^{(p)}$ is an elliptic curve over $K$ defined as in $\S 2.2 .2$ such that $E$ has $B_{K, p^{-}}$ reduction.

3.2.1. The case $\boldsymbol{p}=11$. Let $E=E_{t}^{(11)}, t \in \mathbb{Z}$ be an elliptic curve over $K=\mathbb{Q}\left(\sqrt{d_{11}}\right)$ defined as in $\S 2.2 .2$. A computation shows that the discriminant of $E$ is equal to

$$
\Delta(E)=-\frac{t^{3}+t-2-t \sqrt{d_{11}}}{2} \cdot b_{11}^{3} \cdot T
$$

where

$$
\begin{aligned}
T= & -t^{3}\left(t^{9}+5 t^{8}+6 t^{7}+3 t^{6}-14 t^{5}-14 t^{4}+t^{3}+11 t^{2}-1\right) \\
& +t^{3}\left(t^{7}+5 t^{6}+5 t^{5}-9 t^{3}-6 t^{2}+2 t+1\right) \sqrt{d_{11}} \in \mathcal{O}_{K} .
\end{aligned}
$$

Furthermore, we have $\operatorname{Nm}_{K / \mathbb{Q}}(T)=-4 t^{10} \cdot\left(t^{5}-t^{4}-15 t^{3}+14 t^{2}+3 t-1\right)$. Let $F=t^{5}-t^{4}-15 t^{3}+14 t^{2}+3 t-1 \in \mathbb{Z}$. Since $\Delta(E) \neq 0$, we see $F \neq \pm 1$. Therefore there exists a prime $\ell$ with $\ell \mid F$, and hence the elliptic curve $E$ has bad reduction at some prime $\mathfrak{q}$ of $K$ over $\ell$. We note that the solutions of the equation

$$
X^{5}-X^{4}-15 X^{3}+14 X^{2}+3 X-1=0
$$

define the extension field $\mathbb{Q}\left(\zeta_{11}+\zeta_{11}^{-1}\right)$ over $\mathbb{Q}$. By a similar argument of the proof of Proposition 3.1, we have $\ell \equiv \pm 1 \bmod 11$ if $\ell \neq 11$. Therefore we have the following:

Proposition 3.2. Let $E=E_{t}^{(11)}, t \in \mathbb{Z}$ be an elliptic curve over $K=\mathbb{Q}\left(\sqrt{d_{11}}\right)$ defined as in $\S 2.2 .2$. Then $E$ does not have $B_{K, 11}$-reduction.

3.2.2. The case $\boldsymbol{p}=13$. Let $E=E_{t}^{(13)}, t \in \mathbb{Z}$ be an elliptic curve over $K=\mathbb{Q}\left(\sqrt{d_{13}}\right)$ defined as in $\S 2.2 .2$. A computation shows that the discriminant of $E$ is equal to

$$
\Delta(E)=\frac{t^{4}+t^{3}+t+2-t \sqrt{d_{13}}}{2} \cdot b_{13}^{3} \cdot T,
$$

where $T \in \mathcal{O}_{K}$ satisfies $\operatorname{Nm}_{K / \mathbb{Q}}(T)=4 t^{6} \cdot(t+1)^{3} \cdot\left(t^{3}+4 t^{2}+t-1\right)$. Let $F=t^{3}+4 t^{2}+t-1 \in \mathbb{Z}$. Since $\Delta(E) \neq 0$, we see $F \neq \pm 1$. Therefore there exists a prime $\ell$ with $\ell \mid F$, and hence the elliptic curve $E$ has bad reduction at some prime $\mathfrak{q}$ of $K$ over $\ell$. Let $L$ be the splitting field of the equation

$$
X^{3}+4 X^{2}+X-1=0 .
$$

Then $L$ is a subfield of $\mathbb{Q}\left(\zeta_{13}+\zeta_{13}^{-1}\right)$ with $\left[\mathbb{Q}\left(\zeta_{13}+\zeta_{13}^{-1}\right): L\right]=2$. By a similar argument of the proof of Proposition 3.1, we have $\ell^{2} \equiv \pm 1 \bmod 13$ if $\ell \neq 13$. If there exists a prime $\ell \neq 13$ such that $\ell \mid F$ and the residue degree of $K$ over $\ell$ is equal to 2 , then the elliptic curve $E$ does not have $B_{K, 13}$-reduction. Hence 
we conclude that an elliptic curve $E_{t}^{(13)}, t \in \mathbb{Z}$ does not have $B_{K, 13}$-reduction with very high probability.

\section{Mod $p$ Galois representations}

In this section, we consider the irreducibility and surjectivity of $\bar{\rho}_{E, p}$ for semistable elliptic curves $E$ over a number field $K$ with $\mathfrak{q} \in B_{K, p}$-reduction.

\subsection{Classification of $\mathcal{O}_{K}$-group schemes of order $p$}

Let $K$ be a number field. Fix a prime number $p$. We shall review the classification of $\mathcal{O}_{K}$-group schemes of order $p$ due to Oort and Tate [9]. Let $M$ be the set of non-generic points of $\operatorname{Spec}\left(\mathcal{O}_{K}\right)$, and let $M_{p}$ denote the set of $\mathfrak{p} \in M$ such that $\mathfrak{p}$ divides $p$. Let $F$ denote the functor which associates with each commutative ring $R$ with unity the set $F(R)$ of isomorphism classes of $R$-group schemes of order $p$. Then Oort and Tate showed that the square

$$
\begin{aligned}
& \begin{array}{c}
F\left(\mathcal{O}_{K}\right) \\
\downarrow
\end{array} \rightarrow \prod_{\mathfrak{p} \in M} F\left(\mathcal{O}_{\mathfrak{p}}\right) \\
& F(K) \rightarrow \prod_{\mathfrak{p} \in M} F\left(K_{\mathfrak{p}}\right)
\end{aligned}
$$

is Cartesian (see [9, Lemma 4]). Let $C_{K}$ denote the idèle class group of $K$. Using class field theory, there are canonical bijections

$$
\begin{aligned}
F(K) & \simeq \operatorname{Hom}_{\text {cont }}\left(C_{K}, \mathbb{F}_{p}^{\times}\right), \\
F\left(K_{\mathfrak{p}}\right) & \simeq \operatorname{Hom}_{\text {cont }}\left(K_{\mathfrak{p}}^{\times}, \mathbb{F}_{p}^{\times}\right)(\mathfrak{p} \in M), \text { and } \\
F\left(\mathcal{O}_{\mathfrak{p}}\right) & \simeq \operatorname{Hom}_{\text {cont }}\left(K_{\mathfrak{p}}^{\times} / U_{\mathfrak{p}}, \mathbb{F}_{p}^{\times}\right)\left(\mathfrak{p} \in M \backslash M_{p}\right),
\end{aligned}
$$

where $H_{\text {cont }}$ denotes the set of the continuous homomorphisms and $U_{\mathfrak{p}}$ is the group of units in $\mathcal{O}_{\mathfrak{p}}$ (see [9, Lemma 6]). Via these bijections, the arrows in the above diagram are induced by the canonical homomorphisms $K_{\mathfrak{p}}^{\times} \rightarrow C_{K}$ and $K_{\mathfrak{p}}^{\times} \rightarrow K_{\mathfrak{p}}^{\times} / U_{\mathfrak{p}}$.

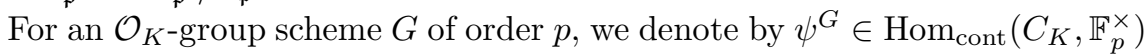
the idèle character determined by $G \otimes K$, and by $\psi_{\mathfrak{p}}^{G}$ the corresponding character of $K_{\mathfrak{p}}^{\times}$for $\mathfrak{p} \in M$. For each $\mathfrak{p} \in M_{p}$, we let $n_{\mathfrak{p}}^{G}=v_{\mathfrak{p}}(a)$, where $v_{\mathfrak{p}}$ is the normalized discrete valuation of $K_{\mathfrak{p}}$ and $a$ is the element of $\mathcal{O}_{\mathfrak{p}}$ such that $G \otimes \mathcal{O}_{\mathfrak{p}} \simeq G_{a, b}$ in the notation of [9]. Note that $n_{\mathfrak{p}}^{G}$ is uniquely determined by $G$. Oort and Tate showed the following [9, Theorem 3]:

Theorem 4.1 (Oort-Tate). The map $G \mapsto\left(\psi^{G},\left(n_{\mathfrak{p}}^{G}\right)_{\mathfrak{p} \in M_{p}}\right)$ gives a bijection between the isomorphism classes of $\mathcal{O}_{K}$-group schemes of order $p$ and the systems $\left(\psi,\left(n_{\mathfrak{p}}\right)_{\mathfrak{p} \in M_{p}}\right)$ consisting of a continuous homomorphism $\psi: C_{K} \rightarrow \mathbb{F}_{p}^{\times}$ and for each $\mathfrak{p} \in M_{p}$ an integer $n_{\mathfrak{p}}$ with $0 \leq n_{\mathfrak{p}} \leq e_{\mathfrak{p}}$, which satisfy the following conditions:

(A) For $\mathfrak{p} \in M \backslash M_{p}, \psi$ is unramified at $\mathfrak{p}$, i.e., $\psi_{\mathfrak{p}}\left(U_{\mathfrak{p}}\right)=1$,

(B) For $\mathfrak{p} \in M_{p}, \psi_{\mathfrak{p}}(u)=\left(\mathrm{Nm}_{k_{\mathfrak{p}} / \mathbb{F}_{\mathfrak{p}}}(\bar{u})\right)^{-n_{\mathfrak{p}}}, \forall u \in U_{\mathfrak{p}}$, where $u \mapsto \bar{u}$ denotes the residue class map. 
Here $\psi_{\mathfrak{p}}: K_{\mathfrak{p}}^{\times} \rightarrow \mathbb{F}_{p}^{\times}$denotes the local character induced by $\psi$ via the canonical $\operatorname{map} K_{\mathfrak{p}}^{\times} \rightarrow C_{K}$.

For a given family $\left(n_{\mathfrak{p}}\right)_{\mathfrak{p} \in M_{p}}$, there is no idèle class character $\psi$ satisfying the conditions (A) and (B) of Theorem 4.1, or the set of all idèle characters is a principal homogeneous space under the group of homomorphisms of the narrow class group of $K$ into $\mathbb{F}_{p}^{\times}$. Therefore, if the narrow class number $h_{K}^{+}$ of $K$ is prime to $(p-1)$, there is at most one $\psi$ for a given family $\left(n_{\mathfrak{p}}\right)_{\mathfrak{p} \in M_{p}}$. Furthermore, for an $\mathcal{O}_{K}$-group scheme $G$ of order $p$ corresponding to $\left(n_{\mathfrak{p}}\right)_{\mathfrak{p} \in M_{p}}$, the Cartier dual of $G$ corresponds to $\left(e_{\mathfrak{p}}-n_{\mathfrak{p}}\right)_{\mathfrak{p} \in M_{p}}$.

\subsection{Irreducibility and surjectivity of $\bar{\rho}_{E, p}$}

Let $p$ be a prime number. Let $E$ be an elliptic curve over a number field $K$. If the representation $\bar{\rho}_{E, p}$ is reducible, then we have

$$
\bar{\rho}_{E, p} \sim\left(\begin{array}{cc}
\phi_{1} & * \\
0 & \phi_{2}
\end{array}\right), \phi_{i}: G_{K} \rightarrow \mathbb{F}_{p}^{\times}, i=1,2
$$

We note that each character $\phi_{i}$ induces $\bar{\phi}_{i}: \operatorname{Gal}\left(K^{\mathrm{ab}} / K\right) \rightarrow \mathbb{F}_{p}^{\times}$, where $K^{\mathrm{ab}}$ is the maximal abelian extension field of $K$.

Proposition 4.2. Assume that $E$ is a semistable elliptic curve with good reduction at the primes of $K$ over $p$. Then each character $\phi_{i}$ gives an $\mathcal{O}_{K}$-group scheme $G$ of order $p$ with $\psi^{G}=\bar{\phi}_{i} \circ \sigma_{K}$, where $\sigma_{K}: C_{K} \rightarrow \operatorname{Gal}\left(K^{\mathrm{ab}} / K\right)$ is the reciprocity map.

Proof. Since $E$ has good reduction at the primes $\mathfrak{p}$ of $K$ over $p$, the $p$-torsion subgroup $E[p]$ is an $\mathcal{O}_{\mathfrak{p}}$-group scheme. Therefore it follows that $\phi_{i}$ has a structure of an $\mathcal{O}_{\mathfrak{p}}$-group scheme of order $p$. Let $S_{E}$ denote the set of the primes of $K$ at which $E$ has bad reduction. Let $\mathfrak{q}$ be a prime of $K$ with $\mathfrak{q} \nmid p$.

- In the case $\mathfrak{q} \notin S_{E}$, the representation $\bar{\rho}_{E, p}$ is unramified at $\mathfrak{q}$ by the criterion of Néron-Ogg-Shafarevich (see [12, Chapter VII, Theorem 7.1]). Hence $\phi_{i}$ is unramified at $\mathfrak{q}$.

- In the case $\mathfrak{q} \in S_{E}$, there exists an unramified extension field $L$ over $K_{\mathfrak{q}}$ of degree 1 or 2 such that $E$ is isomorphic to the Tate curve $E_{q}$ over $L$, where $q$ is the Tate parameter (see $[11, \S 1.12]$ ). By the theory of Tate curves, we have $E(L) \simeq \bar{L}^{\times} / q^{\mathbb{Z}}$. With this identification, we clearly have $E[p] \simeq\left(\zeta_{p} \cdot Q^{\mathbb{Z}}\right) / q^{\mathbb{Z}}$, where $Q=q^{1 / p} \in \bar{L}$ is a fixed $p$-th root of $q$. Therefore the representation $\bar{\rho}_{E, p}$ restricted to $\operatorname{Gal}(\bar{L} / L)$ has the form $\left(\begin{array}{ll}1 & * \\ 0 & \chi\end{array}\right)$, where $\chi$ is the cyclotomic character. Hence it follows that $\phi_{i}$ is unramified at $\mathfrak{q}$.

The above argument shows that $\phi_{i}$ is unramified at the primes $\mathfrak{q}$ of $K$ with $\mathfrak{q} \nmid p$. Therefore we can see that the character $\bar{\phi}_{i} \circ \sigma_{K}$ satisfies the conditions (A) and (B) of Theorem 4.1. This completes the proof. 
Let $K$ be a cubic field. We next consider the structure of $\mathcal{O}_{K}$-group schemes of order $p$. Our result is as follows:

Proposition 4.3. Let $p$ be an odd prime number. Let $K$ be a cubic field with $\operatorname{gcd}\left(p-1, h_{K}^{+}\right)=1$. If $p$ satisfies either the condition $(\mathrm{A})$ or $(\mathrm{B})$ of Theorem 1.2 , then the only $\mathcal{O}_{K}$-group schemes of order $p$ are $\mathbb{Z} / p \mathbb{Z}$ and $\mu_{p}$, where $\mathbb{Z} / p \mathbb{Z}$ (resp. $\left.\mu_{p}\right)$ is a constant (resp. diagonalizable) group scheme.

Proof. In the case where $p$ is prime in $\mathcal{O}_{K}$, this is prove by Oort-Tate [9]. We next consider the case where $p$ is not prime in $\mathcal{O}_{K}$. For simplicity, we only consider the case where $K$ is a complex number field. Assume $p \nmid \mathrm{Nm}_{K / \mathbb{Q}}(u-1)$, where $u$ is a fundamental unit of $K$ such that $u>0$ with respect to the only one real place of $K$.

The basic idea is based on [4]. We first consider the case $p \mathcal{O}_{K}=\mathfrak{p}_{1}^{2} \mathfrak{p}_{2}$, where $\mathfrak{p}_{1}, \mathfrak{p}_{2}$ are the primes of $K$ over $p$. For the family $\left(n_{\mathfrak{p}_{1}}, n_{\mathfrak{p}_{2}}\right)=(1,0)$, we assume that there is an idèle class character $\psi: C_{K} \rightarrow \mathbb{F}_{p}^{\times}$satisfying the conditions (A) and (B) of Theorem 4.1. According to class field theory, there is a modulus of the form $\mathfrak{m}=\infty \cdot \mathfrak{p}_{1}$ such that $\psi$ induces a surjective homomorphism $\bar{\psi}: C_{m} \rightarrow \mathbb{F}_{p}^{\times}$, where $C_{\mathfrak{m}}$ is the ray class group of $K$ modulus $\mathfrak{m}$. Let $C_{\infty}$ be the narrow class group of $K$. There is an exact sequence [8]

$$
1 \rightarrow U^{+} / U_{\mathfrak{m}, 1} \rightarrow\left(\mathcal{O}_{K} / \mathfrak{p}_{1}\right)^{\times} \rightarrow C_{\mathfrak{m}} \rightarrow C_{\infty} \rightarrow 1,
$$

where $U^{+}$is the group of totally positive units of $K$ and $U_{\mathfrak{m}, 1}$ is the subgroup of the elements of $U^{+}$which are congruent to 1 modulo $\mathfrak{p}_{1}$. Since $\operatorname{gcd}\left(p-1, h_{K}^{+}\right)=$ 1 , we see that a composition of the following maps

$$
\Phi:\left(\mathcal{O}_{K} / \mathfrak{p}_{1}\right)^{\times} \rightarrow C_{\mathfrak{m}} \stackrel{\bar{\psi}}{\longrightarrow} \mathbb{F}_{p}^{\times}
$$

is an isomorphism. Since $u \in U^{+}$and the image of $U^{+}$is in the kernel of the isomorphism $\Phi$, we have $u-1 \in \mathfrak{p}_{1}$. This is a contradiction to the condition $p \nmid$ $\mathrm{Nm}_{K / \mathbb{Q}}(u-1)$. Therefore there is no idèle character $\psi$ satisfying the conditions (A) and (B) of Theorem 4.1 for the family $\left(n_{\mathfrak{p}_{1}}, n_{\mathfrak{p}_{2}}\right)=(1,0)$. Furthermore, considering the Cartier dual, the same result holds for the family $\left(n_{\mathfrak{p}_{1}}, n_{\mathfrak{p}_{2}}\right)=$ $(1,1)$. A similar argument shows that there is no idèle class character for families $\left(n_{\mathfrak{p}_{1}}, n_{\mathfrak{p}_{2}}\right)=(0,1),(2,0)$. Therefore the only $\mathcal{O}_{K}$-group schemes of order $p$ are $\mathbb{Z} / p \mathbb{Z}$ and $\mu_{p}$ by Theorem 4.1 .

In the case where $p \mathcal{O}_{K}=\mathfrak{p}^{3}, \mathfrak{p}_{1} \mathfrak{p}_{2} \mathfrak{p}_{3}$ or $\mathfrak{p}_{1} \mathfrak{p}_{2}$, a similar argument as above shows the same result. This completes the proof.

We note that Theorem 1.1 holds for the primes $p \geq 3$ when $E$ is restricted to be semistable. Combining Propositions 4.2 and 4.3 with Theorem 1.1, we can prove Theorem 1.2 as follows:

Proof of Theorem 1.2. The idea is based on the proof of [7, Theorem 4] or [4, Théorème]. Suppose that $\bar{\rho}_{E, p}$ is reducible. Then $\bar{\rho}_{E, p}$ has the form (9). Since $\phi_{1} \cdot \phi_{2}=\chi$, it follows from Propositions 4.2 and 4.3 that either of $\phi_{i}$ is a trivial character. Hence we see that $E$ or $E^{\prime}=E / \mu_{p}$ has a $p$-torsion point. Since $E$ 
and $E^{\prime}$ have bad reduction at same primes, this is a contradiction to Theorem 1.1. This completes the proof of Theorem 1.2.

Example. Let $K$ be a cubic field with $h_{K}^{+}=1$. Let $E$ be an elliptic curve over $K$ with everywhere good reduction. By Theorem 1.2, we obtain the following results on the irreducibility of $\bar{\rho}_{E, p}$ :

- Let $K=\mathbb{Q}[x] /(f(x))$ be a complex cubic field defined by $f(x)=x^{3}+$ $x-1$. We have that the element $x \in \mathcal{O}_{K}$ is a fundamental unit of $K$ with $x>0$ and $\operatorname{Nm}_{K / \mathbb{Q}}(x-1)=1$. We also have $3 \mathcal{O}_{K}=\mathfrak{p}_{1} \mathfrak{p}_{2}$ with $f_{1}=1$ and $f_{2}=2$, where $f_{i}$ is the residue degree of $\mathfrak{p}_{i}$. Since $\sharp E\left(k_{\mathfrak{p}_{1}}\right) \leq 3+1+2 \sqrt{3}<8$, the elliptic curve $E$ has no $p$-torsion points for the primes $p \geq 11$. By a similar argument of the proof of Theorem 1.2 , we see that $\bar{\rho}_{E, p}$ is irreducible for the primes $p \geq 11$. Furthermore, since $h_{K\left(\zeta_{p}\right)}=1$ for $p=3,5$ and 7 , it follows from Theorem 1.2 that $\bar{\rho}_{E, p}$ is irreducible for $p=3,5$ and 7 . Therefore we have that $\bar{\rho}_{E, p}$ is irreducible for the primes $p \geq 3$.

- Let $K=\mathbb{Q}[x] /(f(x))$ be a totally real cubic field defined by $f(x)=$ $x^{3}-x^{2}-3 x-1$. We have that elements $x, x^{2}-2 x \in \mathcal{O}_{K}$ are two independent fundamental units of $K$ with $\mathrm{Nm}_{K / \mathbb{Q}}\left(x^{2}-1\right)=4$ and $\mathrm{Nm}_{K / \mathbb{Q}}\left(\left(x^{2}-2 x\right)^{2}-1\right)=16$. We also have $2 \mathcal{O}_{K}=\mathfrak{p}^{3}$. Since $\sharp E\left(k_{\mathfrak{p}}\right) \leq$ $2+1+2 \sqrt{2}<7$, the elliptic curve $E$ has no $p$-torsion points for the primes $p \geq 7$. By a similar argument of the proof of Theorem 1.2, we see that $\bar{\rho}_{E, p}$ is irreducible for the primes $p \geq 7$. Furthermore, since $h_{K\left(\zeta_{3}\right)}=1$ and $h_{K\left(\zeta_{5}\right)}=2$, it follows from Theorem 1.2 that $\bar{\rho}_{E, p}$ is irreducible for $p=3$ and 5 . Therefore we have that $\bar{\rho}_{E, p}$ is irreducible for the primes $p \geq 3$.

We give the following result mainly due to Serre [11]:

Proposition 4.4. Let $K$ be a number field with $h_{K}^{+}=1$ and let $D_{K}$ denote the discriminant of $K$. Let $E$ be a semistable elliptic curve over $K$ with the $j$-invariant $j_{E}$. Let $p$ be a prime such that $p \nmid D_{K}$. If $p=2,3$ or 5 , suppose $p \nmid v\left(j_{E}\right)$ for some $v \in S_{E}$, where $S_{E}$ is the set of the finite places $v$ at which $E$ has bad reduction. If $\bar{\rho}_{E, p}$ is irreducible, then $\bar{\rho}_{E, p}$ is surjective.

Proof. See the proof of [11, Proposition 21] or [4, §3].

Combining the above result with Theorem 1.2, we obtain the following result on the surjectivity of $\bar{\rho}_{E, p}$.

Theorem 4.5. Let $K$ be a cubic field with $h_{K}^{+}=1$ and let $D_{K}$ denote the discriminant of $K$. Let $p$ be an odd prime number such that $p \nmid D_{K}$. Suppose that $p \nmid h_{K\left(\zeta_{p}\right)}$ and $e_{\mathfrak{p}}<p-1$ for the primes $\mathfrak{p}$ of $K$ over $p$. Let $E$ be a semistable elliptic curve over $K$ with $B_{K, p}$-reduction. If $p=3$ or 5 , suppose $p \nmid v\left(j_{E}\right)$ for some $v \in S_{E}$. If $p$ satisfies either the condition (A) or (B) of Theorem 1.2, then $\bar{\rho}_{E, p}$ is surjective. 
Remark. In the case where $K=\mathbb{Q}$ or $K$ is a quadratic field, we can show similar results of Theorems 1.2 and 4.5 (see also [4] in the case where $K$ is a quadratic field). However, in general, it does not hold in the case $[K: \mathbb{Q}] \geq 4$.

\section{3. $K\left(\zeta_{p}\right)$-rational points of order $p$}

Let $K$ be a number field. Let $E$ be an elliptic curve over $K$ with a $p$-torsion point. There exist an elliptic curve $E^{*}$ over $K$ and a $K$-isogeny $E \rightarrow E^{*}$ with kernel $\mathbb{Z} / p \mathbb{Z}$. By the exact sequence $(1)$, we have $\mu_{p} \subset E^{*}$ and hence $E^{*}$ has a $K\left(\zeta_{p}\right)$-rational point of order $p$. For an elliptic curve $E$ over $K$ with $B_{K, p^{-}}$ reduction, we here consider the existence of a $K\left(\zeta_{p}\right)$-rational point of $E$ of order $p$. By Theorem 1.2, we obtain the following result (cf. Theorem 1.1):

Proposition 4.6. Let $p$ be an odd prime number. Let $K$ be a cubic field with $\operatorname{gcd}\left(p-1, h_{K}^{+}\right)=1$. Suppose that $p \nmid K\left(\zeta_{p}\right)$ and $e_{\mathfrak{p}}<p-1$ for the primes $\mathfrak{p}$ of $K$ over $p$. Let $E$ be a semistable elliptic curve over $K$ with $B_{K, p}$-reduction. If $p$ satisfies either $(\mathrm{A})$ or $(\mathrm{B})$ of Theorem 1.2, then $E$ has no $K\left(\zeta_{p}\right)$-rational points of order $p$.

Proof. Suppose that $E$ has a $K\left(\zeta_{p}\right)$-rational point of order $p$. Set $L=K(E[p])$ and $M=K\left(\zeta_{p}\right)$. Let $G$ be a simple subgroup of $E[p]$ as a $\operatorname{Gal}(L / K)$-module. Set $S_{p}=\operatorname{Gal}(L / M)$. We may assume that $S_{p}$ is non-trivial. Then the order of $S_{p}$ is equal to $p$. Since $S_{p} \triangleleft \operatorname{Gal}(L / K)$, we see that the set $G(\bar{K})^{S_{p}}$ of the $S_{p}$-fixed points is a $\operatorname{Gal}(L / K)$-submodule of $G(\bar{K})$. Since $\sharp G(\bar{K}) \equiv \sharp G(\bar{K})^{S_{p}} \bmod p$, the group $G(\bar{K})^{S_{p}}$ is non-trivial, and hence $G(\bar{K})=G(\bar{K})^{S_{p}}$ because $G$ is simple. Then $G(\bar{K})$ is a $\operatorname{Gal}(M / K)$-module. Since the group $\operatorname{Gal}(M / K)$ has exponent dividing $p-1$, the $\mathbb{F}_{p}[\operatorname{Gal}(M / K)]$-module $G(\bar{K})$ is a product of 1-dimensional eigenspaces. Since $G$ is simple, there is only one such eigenspace, and hence $G$ has order $p$. Therefore the representation $\bar{\rho}_{E, p}$ is reducible. But this contradicts to Theorem 1.2. This completes the proof of Proposition 4.6.

\section{References}

[1] D. Jeon, C. H. Kim, and Y. Lee, Families of elliptic curves over quartic number fields with prescribed torsion subgroups, Math. Comp. 80 (2011), no. 276, 2395-2410.

[2] S. Kamienny, Torsion points on elliptic curves and q-coefficients of modular forms, Invent. Math. 109 (1992), no. 2, 221-229.

[3] M. Kenku and F. Momose, Torsion points on elliptic curves defined over quadratic fields, Nagoya Math. J. 109 (1988), 125-149.

[4] A. Kraus, Courbes elliptiques semi-stables et corps quadratiques, J. Number Theory 60 (1996), no. 2, 245-253.

[5] D. Kubert, Universal bounds on the torsion of elliptic curves, Proc. London Math. Soc. (3) 33 (1976), no. 2, 193-237.

[6] B. Mazur, Modular curves and the Eisenstein ideal, Inst. Hautes Études Sci. Publ. Math. 47 (1977), 33-186.

[7] _ Rational isogenies of prime degree, Invent. Math. 44 (1978), no. 2, 129-162.

[8] J. Neukirch, Algebraic Number Theory, Grudlehren der Mathematischen Wisenshaften, vol. 322, Springer-Verlag, Berlin-Heidelberg New York, 1999. 
[9] Oort and Tate, Group schemes of prime order, Ann. Sci. École Norm. Sup. 3 (1970), $1-21$.

[10] The PARI Group, Bordeaux, PARI/GP, available from http://pari.math.u-bordeaux. fr/doc.html.

[11] J.-P. Serre, Propriétés galoisiennes des points d'ortre fini des courbes elliptiques, Invent. Math. 15 (1972), no. 4, 259-331.

[12] J. H. Silverman, The Arithmetic of Elliptic Curves, Graduate Texts in Math. 106, Springer-Verlag, Berlin-Heidelberg New York, 1994.

[13] L. C. Washington, Introduction to Cyclotomic Fields, Graduate Texts in Math. 83, Springer-Verlag, Berlin-Heidelberg New York, 1982.

[14] M. Yasuda, Torsion points of elliptic curves with bad reduction at some primes, to appear in Commentarii Math. Univ. St. Pauli.

Fujitsu Laboratories LTD.

4-1-1, Kamikodanaka, Nakahara-ku, KaWasaki

211-8588, JAPAN

E-mail address: myasuda@labs.fujitsu.com 